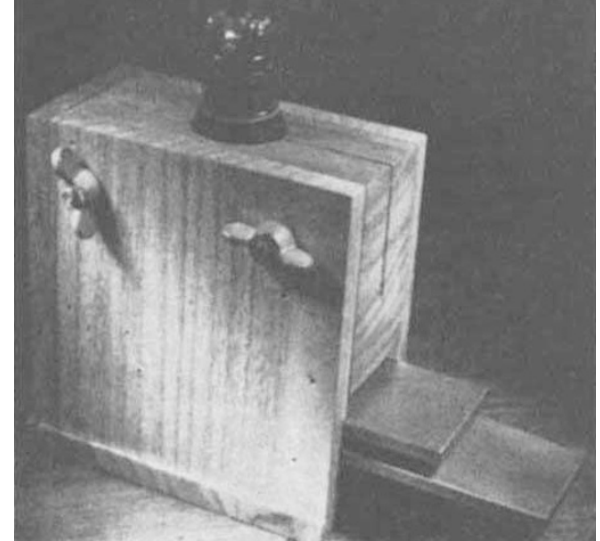

Fig. 1

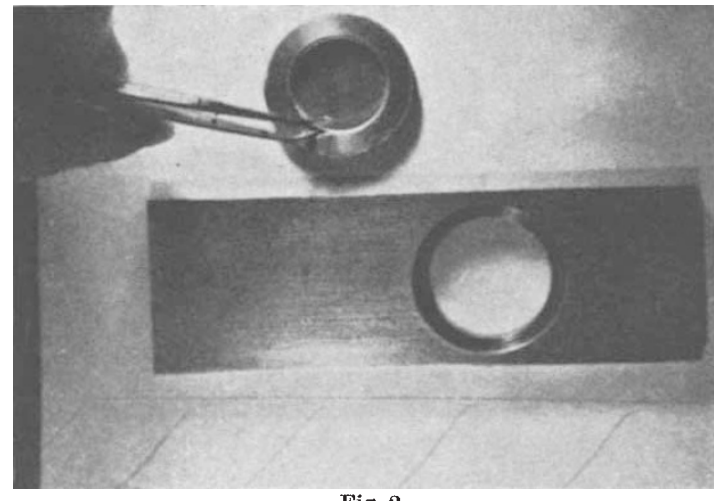

Fig. 2

also as a uniform back scatterer if the castle is moved from one table to another. The rear serves as a stop so that all the samples are uniformly aligned beneath the Geiger-Müller tube. The sides are grooved to carry the sample holders. The number of grooves and their distance apart can be suited to the user.

The sample supports (Fig. 2) were made on a metal lathe using stock steel $\frac{1}{4}$ in. thick and 21 in. wide. A shelf was left at the bottom just large enough to support the steel ring which fits inside. This ring carries the sample pans with the radioactive material. The ring has a groove to make the handling of the pans easier. The convenience of the ring, which was made from bar steel, lies not only in the fact that it can be made to fit any size sample pan, but also it is excellent when a non back-scattering support is required. Fig. 3 shows a piece of thin 'Pliofilm' stuck

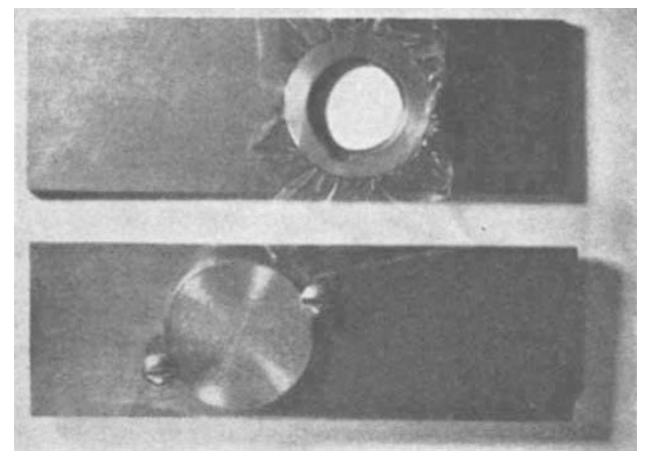

Fig. 3 between the ring and the support. The bottom photograph shows how back scatterers can easily be held in place.

The geometry has been reproducible with no difficulty. The wooden castle and two steel holders were made for about 5.00 American dollars.

The background using a 900-volt Geiger-Müller tube with a mica window of thickness $1.4 \mathrm{mgm} . / \mathrm{cm}$.? is about 70 counts $/ \mathrm{min}$. at an altitude of $600 \mathrm{~m}$.

This work was supported by a U.S. Atomic Energy Commission Contract $A T(30-1) 2043$.

Howard Boroughs

Inter-American Institute of Agricultural Sciences, Turrialba, Costa Rica.

\section{Time-lags in the Intrinsic Electrical Breakdown of Glass}

WHEN measuring the electrical breakdown strength of lead glass sheets with 1-cm.-diam. sphere electrodes subjected to $0 \cdot 1 / 1,500 \mu$ sec. voltage pulses it was found that the secondary effects, usually present when transformer oil or similar immersion media are used, could be eliminated by using low-conductivity water as the immersion medium. Under these con ditions when the edge effects were suppressed it was observed that the breakdown took place after a time delay in the range of $10^{-7}-10^{-4}$ sec. The distribution of these time delays was found to be random. The results fit the relationship :

$$
n / n_{0}=\exp (-t / \tau)
$$

where $n$ is the number of observed time lags within any time $t ; n_{0}$ is the total number of time lags observed; $\tau$ is a constant.

These results are comparable with the findings of Cooper and Grossart ${ }^{1}$ on potassium chloride erystals, polythene and polystyrene and the experiments done by Kawamura ${ }^{2-4}$ and his collaborators on mica.

The intrinsic electric strength of lead glass under low-conductivity water was found to be $\mathbf{1 1 \cdot 4} \pm \mathbf{l} \cdot \mathbf{1}$ $\times 10^{6} \mathrm{~V} . / \mathrm{cm}$. This is considerably higher than $6 \times 10^{6} \mathrm{~V} . / \mathrm{cm}$. reported by Austen and Whitehead ${ }^{5}$. This work will be fully reported elsewhere.

M. N. Azam

H. Dickinsor

Engineering Department,

University College of Swansea, University of Wales.

${ }^{1}$ Cooper, R., and Grossart, D. T., Proc. Phys. Soc., B, 69, 1351 (1956). ${ }^{2}$ Kawamura, H., Ohkura, H., and Kikuchi, T., J. Phys. Soc. Japan, 9, 541 (1959).

${ }^{3}$ Kawamura, H. Onuki, and Ohkura, H., J. Phys. Soc. Japan, 8, 424 (1953).

4 Kawamura, H. Onuki, J. Phys. Soc. Japan, 8, 731 (1953).

${ }^{5}$ Austen, A. E. W., and Whitehead, S., Proc. Roy. S'oc., A, 176, 33 $(1940)$.

\section{METALLURGY}

\section{Piling-up of Dislocations beneath a Surface Film}

The concept of a surface film obstructing the egress of dislocations from a crystal and thereby causing them to pile-up beneath the film has proved useful in explaining the results of several metallurgical experiments ${ }^{1,2}$. However, direct evidence for such a phenomenon has been lacking. During an investigation to study some aspects of the effects of surface coatings-oxides, metallic and surface-active films, 